\title{
Systemic Inflammatory Response Syndrome Score at Admission Predicts Injury Severity, Organ Damage and Serum Neutrophil Elastase Production in Trauma Patients
}

\author{
Yuichiro Sakamoto ${ }^{1,2}$, Kunihiro Mashiko ${ }^{1,2}$, Hisashi Matsumoto ${ }^{1,2}$, \\ Yoshiaki Hara $^{1,2}$, Noriyoshi Kutsukata ${ }^{1,2}$ and Hiroyuki Yokota ${ }^{1}$ \\ ${ }^{1}$ Department of Emergency and Critical Care Medicine, Graduate School of Medicine, Nippon Medical School \\ ${ }^{2}$ Department of Emergency and Critical Care Medicine, Nippon Medical School Chiba Hokusoh Hospital
}

\begin{abstract}
Background: Systemic inflammatory response syndrome (SIRS) is a clinical condition representing the culmination of the activation of a complex network of acute endogenous mediators.

Materials and Methods: We investigated both the relationship between the results of SIRS assessments in 212 trauma patients at the time of hospital arrival and measures of trauma severity determined using the Injury Severity Score (ISS) and the Revised Trauma Score (RTS). We then considered the possibility of whether this assessment could be used to predict the development of organ dysfunction as a complication in trauma patients after admission. The serum neutrophil elastase (SNE) level was also measured in 47 cases.

Results: The cases with SIRS had a significantly higher ISS and a lower RTS. Organ dysfunction occurred in 22 cases, and a significant correlation was noted between the development of organ dysfunction and the presence of SIRS $(86.4 \%$; 19 cases $/ 22$ cases, $\mathrm{p}=$ $0.0007)$ at the time of arrival. The SNE level was significantly higher among the patients who fulfilled the four SIRS criteria than among the other patients $(\mathrm{p}=0.0301)$.

Conclusion: We concluded that the greater the SIRS score at the time of hospital arrival, the greater the anatomical and physiological severity of the trauma patient's condition.

(J Nippon Med Sch 2010; 77: 138-144)
\end{abstract}

Key words: systemic inflammatory response syndrome (SIRS), Injury Severity Score (ISS), Revised Trauma Score (RTS), probability of survival (Ps), neutrophil elastase, trauma

\section{Introduction}

The definition of systemic inflammatory response syndrome (SIRS) is widely accepted, and was outlined at the joint conference of the American College of Chest Physicians and the Society of Critical Care Medicine in $1992^{12}$. Many of the

Correspondence to Yuichiro Sakamoto, Department of Emergency and Critical Care Medicine, Nippon Medical School Chiba Hokusoh Hospital, 1715 Kamagari, Inzai, Chiba 270-1694, Japan

E-mail: y-sakamoto@nms.ac.jp

Journal Website (http://www.nms.ac.jp/jnms/) 
Table 1 Characteristics of Cases

\begin{tabular}{lc} 
Sex (male/female) & $154(72.6 \%) / 58(27.4 \%)$ \\
Age & $48 \pm 22$ years \\
Body temperature & $36.2 \pm 1.1{ }^{\circ} \mathrm{C}$ \\
Heart rate & $87.9 \pm 21.5 / \mathrm{min}$ \\
Respiratory rate & $24.4 \pm 7.3 / \mathrm{min}$ \\
White blood cell & $12,023 \pm 6,448 / \mathrm{mm}^{3}$ \\
ISS & $16.7 \pm 9.4$ \\
RTS & $7.22 \pm 1.00$ \\
Ps & $0.91 \pm 0.16$ \\
\hline
\end{tabular}

publications on SIRS concern the usefulness of the diagnosis of this syndrome as an early warning sign of sepsis in surgical patients ${ }^{3-8}$. SIRS is a clinical condition that represents the culmination of the activation of a complex network of acute endogenous mediators, namely, inflammatory cytokines. SIRS is not necessarily associated with infections or sepsis, and is also observed in cases of trauma, pancreatitis and burns ${ }^{9-11}$. Thus, SIRS is triggered by an acute reaction in the body and is a sign of poor homeostasis.

Approximately $70 \%$ of patients requiring intensive care meet all the diagnostic criteria for SIRS, and SIRS is reportedly correlated with a poor patient outcome $e^{12-14}$.

We investigated the relationship between the SIRS score on admission and other severity scores such as ISS, RTS and Ps. Then, we clarified whether the SIRS score on admission is useful for predicting the development of organ dysfunction (acute lung injury, disseminated intravascular coagulation or DIC, and/or acute renal failure) as a complication in trauma patients after admission.

\section{Materials and Methods}

Among the 267 patients admitted to our intensive care units or high care units between April 2005 and March 2006, a retrospective analysis was performed in 212 trauma patients. Patients with cardiopulmonary arrest on arrival and those with insufficient data were excluded from the study.

The SIRS score was assessed at the time of admission in all the patients. The ISS, RTS and SIRS score assessments were conducted based on data obtained from the Japan Trauma Databank.
A diagnosis of acute lung injury was made when the $\mathrm{P} / \mathrm{F}$ ratio was 300 or less, and a diagnosis of disseminated intravascular coagulation (DIC) was made when the platelet count was less than 80,000 / $\mathrm{mm}^{3}$ or the serum FDP level was over $25 \mathrm{ug} / \mathrm{mL}$. In addition, a diagnosis of acute renal failure was made when the introduction of temporary hemodiafiltration or the continuous administration of diuretics for over three days was necessary because of a decreased urinary volume.

Statistical analyses were performed using the Jonckheere trend test, the Cochran-Armitage trend test, the t-test and the chi-squared test; a value of $\mathrm{P}<0.05$ was considered statistically significant.

\section{Results}

Two hundred and twelve patients (154 men and 58 women) with trauma were investigated. Blunt trauma was observed in 202 (95.3\%) cases, and penetrating trauma was observed in only 10 cases. The mean patient age was $48 \pm 22$ years. The ISS, RTS and Ps scores were $16.7 \pm 9.4,7.22 \pm 1.0$, and $0.91 \pm 0.16$, respectively (Table $\mathbf{1}$ ).

The proportions of patients fulfilling each of the criteria for the diagnosis of SIRS were as follows: 35.4\% (75 cases) for body temperature, 35.0\% (74 cases) for heart rate, $65.1 \%$ (138 cases) for respiratory rate, and $43.4 \%$ (92 cases) for white blood cell count.

\section{Prevalence of SIRS in Trauma Cases}

The presence of SIRS was diagnosed at the time of admission in 113 out of 212 patients (53.3\%); on the other hand, $13.2 \%$ (28 cases) of the cases did not fulfill any of the diagnostic criteria for SIRS, and $33.5 \%$ (71 cases) fulfilled only one criterion.

\section{SIRS Score and the ISS, RTS and Ps}

A significant relationship between the SIRS score and the ISS, which represents the anatomic severity of a case, was observed in a trend analysis: the greater the number of SIRS criteria fulfilled at the time of hospital arrival, the higher the ISS score (Fig. 1). Similarly, a trend analysis for the RTS, which represents the physiological severity of the 


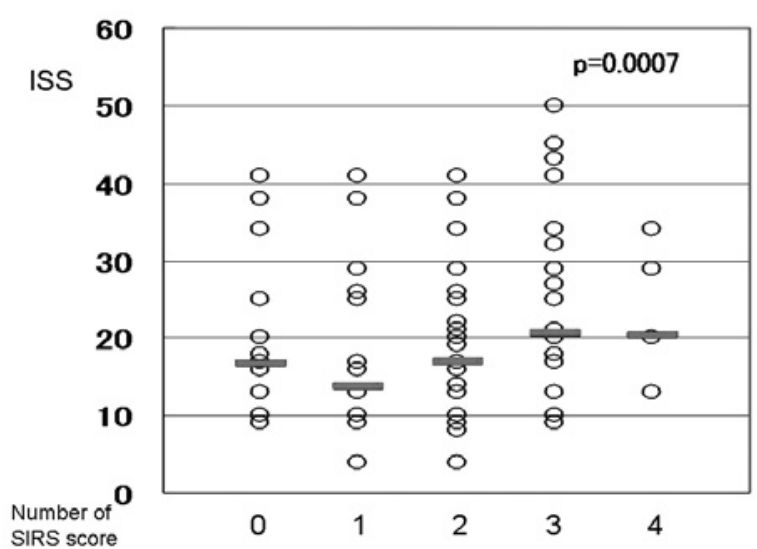

Fig. 1 Relationship between SIRS score and ISS. A significant correlation between the SIRS score upon admission and the ISS was observed $(\mathrm{p}=0.0007)$.

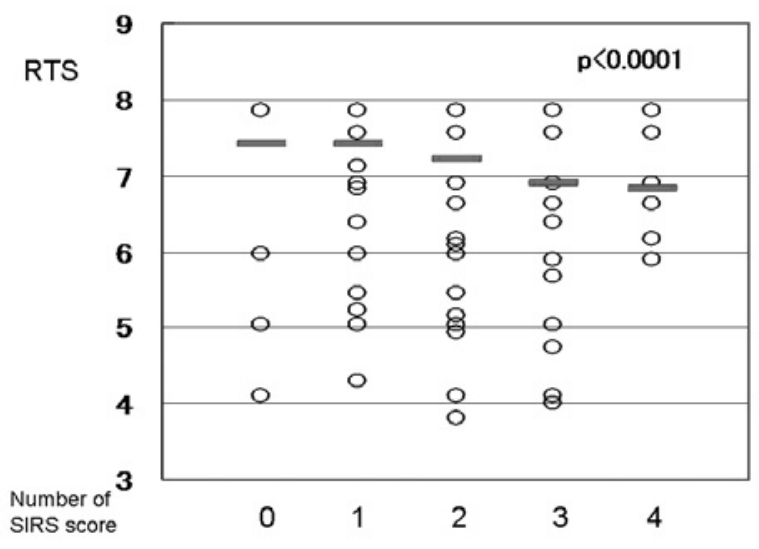

Fig. 2 Relationship between SIRS score and RTS. A significant correlation between the SIRS score upon admission and the RTS was observed $(\mathrm{p}<0.0001)$.

condition of a patient, revealed a statistically significant trend: the greater the number of SIRS criteria fulfilled at the time of hospital arrival, the lower the RTS score (Fig. 2). However, no correlation was observed between the number of SIRS criteria fulfilled at the time of hospital arrival and the Ps (Fig. 3).

An analysis of the relationship between the RTS and SIRS scores revealed that as the SIRS score increased, the percentage of patients with a lessthan-maximum RTS score (7.8408) increased $(\mathrm{p}<$ 0.0001). Among the cases in which at least three of the four SIRS criteria were fulfilled, more than $70 \%$ had an RTS of less than 7.8408.

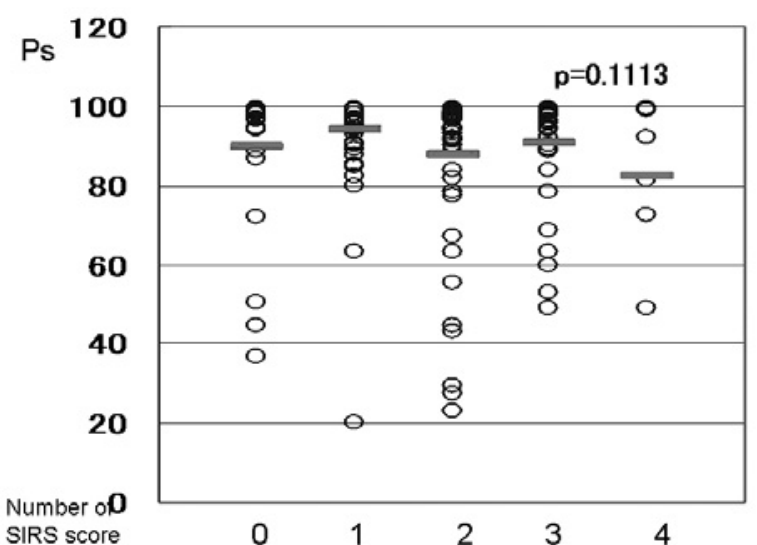

Fig. 3 Relationship between SIRS score and Ps. No correlation between the number of SIRS diagnostic criteria fulfilled at the time of hospital arrival and the projected probability of survival (Ps) was observed.

Relationship between the Diagnosis of SIRS at Admission and the ISS, RTS and Ps

The patients with SIRS showed a significantly higher ISS and a significantly lower RTS (Fig. 4). Although the Ps did not differ significantly between the SIRS and non-SIRS groups at admission, a tendency towards a lower Ps value was observed in the SIRS group (Fig. 4). An analysis of the relationship between the diagnosis of SIRS at admission and the RTS revealed that approximately $60 \%$ of the cases with SIRS at admission had an RTS of less than 7.8408, suggesting the existence of some type of physiological derangement, even when the RTS scores were taken into consideration $(p=0.0008)$.

\section{SIRS and Organ Dysfunction}

Overall, 22 cases developed organ dysfunction; of these cases, $86.4 \% \quad(19 / 22 \quad \mathrm{p}=0.0007)$ had been diagnosed at admission as having SIRS. Specifically, SIRS was diagnosed at admission in $12 / 15$ cases of acute respiratory failure $(80.0 \%), 6 / 9$ cases of renal failure (66.7\%) and $12 / 14$ cases of DIC (85.7\%). Significant correlations were noted between the development of each type of organ dysfunction and the presence of SIRS at admission (Table 2).

SIRS Score and Serum Neutrophil Elastase (SNE) Level

The relationship between the SIRS score and the 

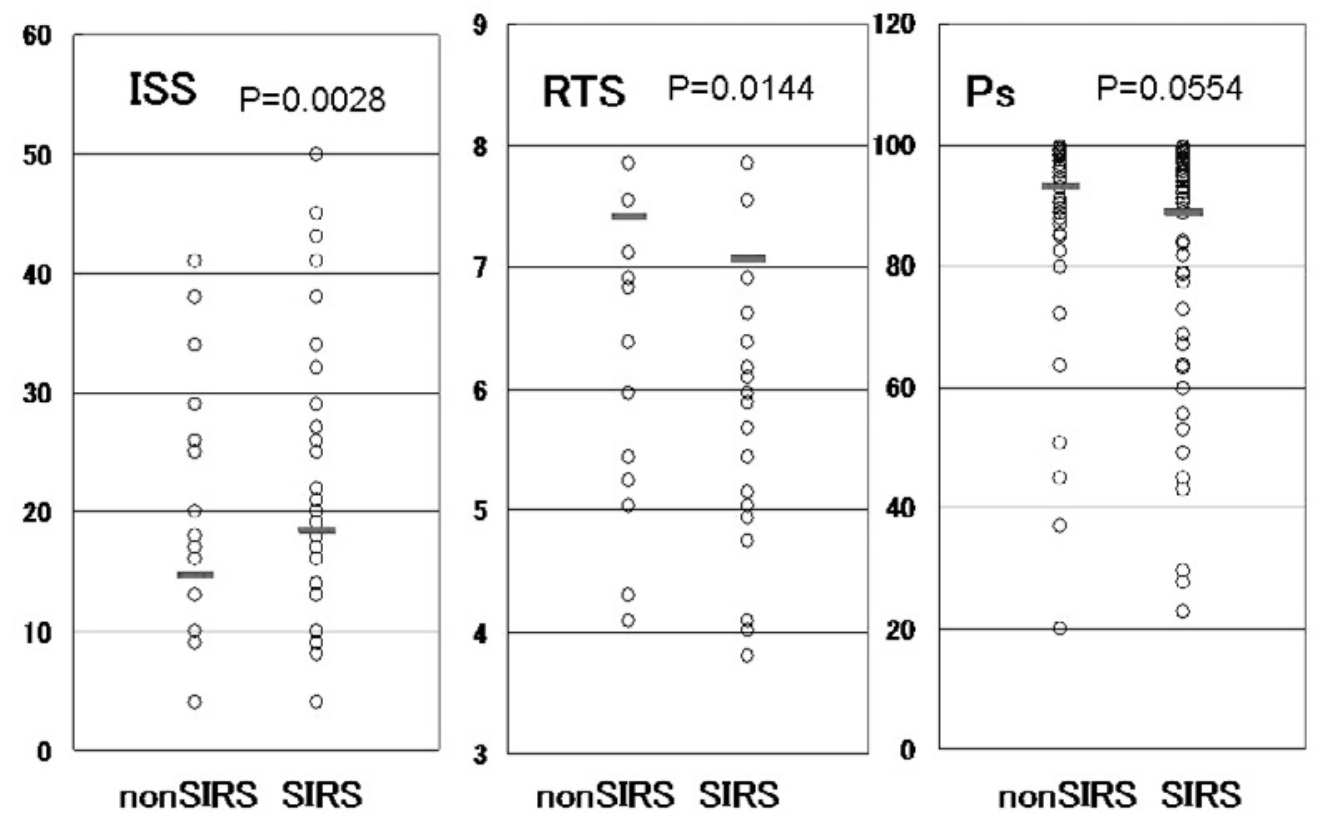

Fig. 4 Relationship between development of SIRS upon admission and ISS, RTS and Ps. A significant difference in the ISS was observed between the non-SIRS and the SIRS groups. A significant difference in the RTS between the two groups was also observed. On the other hand, no significant difference in the Ps between the two groups was seen.

Table 2 Relationship between SIRS and organ dysfunction

\begin{tabular}{lrrr}
\hline & SIRS & non SIRS & $\mathrm{p}$ value \\
\hline Number of case & 113 & 99 & \\
Organ dysfunction & 19 & 3 & 0.0007 \\
Acute respiratory failure & 12 & 3 & 0.0331 \\
DIC & 12 & 2 & 0.0125 \\
Acute renal failure & 6 & 3 & 0.4202 \\
\hline
\end{tabular}

serum neutrophil elastase (SNE) level is shown in Figure 5. The mean SNE level of the patients who fulfilled all four SIRS criteria was $364.3 \mathrm{ng} / \mathrm{mL}$. On the other hand, the mean SNE level was $106.6 \mathrm{ng} /$ $\mathrm{mL}$ among the patients who did not fulfill any of the SIRS criteria, $184.2 \mathrm{ng} / \mathrm{mL}$ among those patients who fulfilled one criterion, $145.6 \mathrm{ng} / \mathrm{mL}$ among those who fulfilled two criteria, and $191.8 \mathrm{ng} / \mathrm{mL}$ among those who fulfilled three criteria. The mean SNE level of the patients who fulfilled all four SIRS criteria was significantly higher than the SNE level among the other patients ( $\mathrm{p}=0.0301$ ) (Fig. 5).

\section{Discussion}

After the excessive biological reactions that can

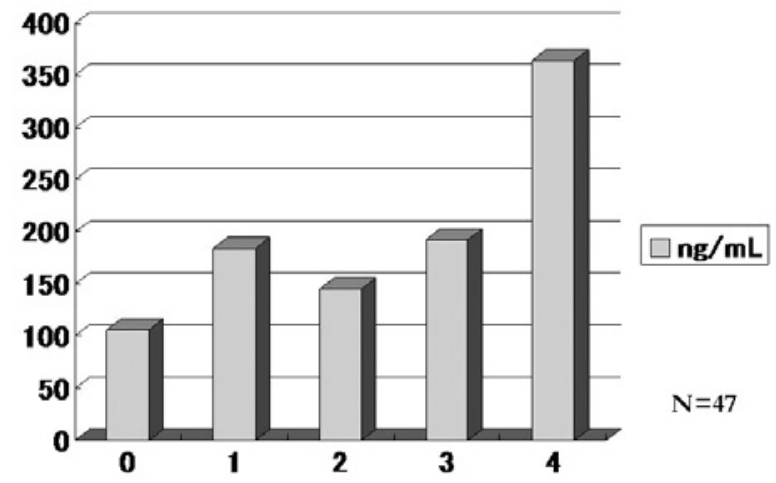

Fig. 5 SIRS score and serum neutrophil elastase (SNE) level. The mean SNE values were $106.6 \mathrm{ng} / \mathrm{mL}$ for the cases that did not fulfill any of the SIRS diagnostic criteria, 184.2 $\mathrm{ng} / \mathrm{mL}$ for those that fulfilled one criteria, $145.6 \mathrm{ng} / \mathrm{mL}$ for those that fulfilled two criteria, $191.8 \mathrm{ng} / \mathrm{mL}$ for those that fulfilled three criteria, and $364.3 \mathrm{ng} / \mathrm{mL}$ for those that fulfilled four criteria.

arise as a result of various insults to the body came to be defined by the term SIRS, hypercytokinemia was reported to be a possible component in the pathogenesis of SIRS ${ }^{15}$. Increases in the levels of inflammatory cytokines are known to occur both locally and systemically in cases with SIRS, and these increases are also associated with a parallel 
increase in the levels of anti-inflammatory cytokines $^{15,16}$.

As far as disease-specific reports on the usefulness of the diagnosis of SIRS are concerned, one report has described a correlation between the presence of SIRS and intestinal necrosis as a result of strangulation ileus ${ }^{17}$, while another report has discussed the usefulness of the diagnosis of SIRS as a factor for predicting the outcome of acute subdural hematoma ${ }^{18}$.

In addition, SIRS is also frequently observed among trauma patients, and the existence of correlations between the SIRS score and the outcome of the patients, as well as the duration of hospitalization, has been reported ${ }^{19}$. In addition, the usefulness of SIRS as a factor for predicting the need for admission to the ICU or the risk of developing infections has also been reported ${ }^{20}$.

Moreover, a recent report of a survey conducted over a three-week post-trauma period provided detailed information on the relationship between post-trauma infections and SIRS, and revealed that the presence of SIRS during the second to third week of hospitalization was a better predictive factor for the development of an infection than the presence of SIRS during the first week ${ }^{21}$.

In trauma patients, various vital reactions are triggered by different injuries, such as ischemic damage arising from hemorrhagic shock, direct damage to tissues and organs arising from contusions, and damage to the central nervous system, such as cerebral contusions and spinal cord injuries.

However, the diagnosis of infections based only on an assessment of the SIRS scores is not realistic. In practice, the presence of infections is normally based on clinical findings and various test results. Various attempts to diagnose infectious SIRS by combining the SIRS assessment with data from other biomarkers have been made. Miller et al. have shown a connection between the SIRS score and the serum CRP with infections in trauma patients admitted to the $\mathrm{ICU}^{20}$. Furthermore, a recent review reported the usefulness of test data, such as the serum CRP, procalcitonin (PCT), neopterin and endotoxin levels, in distinguishing infectious from non-infectious SIRS ${ }^{22,23}$.

Although sporadic reports have described a link between the risk of infections and the presence of SIRS in trauma cases, no reports on the degree of severity of the trauma itself and the presence of SIRS have been made. Therefore, we examined the relationship between SIRS and damage to the body arising from trauma. Specifically, we investigated the correlation between the SIRS score and ISS, which describes the anatomic severity of a trauma case, as well as that between the SIRS score and RTS, which describes the physiological severity of a trauma case. The results showed that as the SIRS score at the time of arrival increased, the anatomic and physiological severity of the trauma case also increased. Correlations were also found between the SIRS assessment and each of the anatomic and physiological variables determining the severity.

The above results suggested the possibility that SIRS assessment at the time of arrival could be converted into a simple score that could represent the overall severity of a case by additionally incorporating the anatomic and physiological severity of the trauma case. In the future, SIRS assessments, when combined with various other test data, may become available as a potential supplementary diagnostic criterion for determining appropriate treatment in trauma cases.

One of the most important factors for determining the outcome of a trauma case is organ dysfunction, which can occur after admission. In the current study, a correlation was recognized between the presence of SIRS upon hospital arrival and the development of acute lung injury and DIC after admission was recognized, suggesting that SIRS assessments are extremely important for improving the outcome of trauma patients. In other words, as in past reports on post-surgical patient cases with prolonged SIRS who exhibited a higher rate of complications and poorer outcomes, it appears that trauma patients diagnosed with SIRS at the time of hospital arrival should be followed up with careful consideration of the possibility that they may develop organ dysfunction. In addition, the SIRS score at the time of admission may be considered as a valuable supplementary diagnostic criterion for the 
early identification of patients at a high risk of developing multiple organ failure.

A recent review concluded that the neutrophil elastase levels are increased in clinical and animal models of acute lung injury, and inhibition of the neutrophil elastase activity levels reduces the symptoms of acute lung injury ${ }^{24}$. Another paper showed close correlations among neutrophilendothelial cell interactions, disseminated intravascular coagulation (DIC), and multiple organ dysfunction syndrome (MODS) in patients with sepsis, severe sepsis and septic shock ${ }^{25}$.

The SNE level cannot be measured easily and rapidly, and in this study, trauma cases with a maximum SIRS score at the time of admission showed high SNE levels. Therefore, the trauma severity scores, such as ISS and RTS, of the patients with a maximum SIRS score were more severe than those who fulfilled only three criteria. Thus, a maximum SIRS score may be a reliable indicator of a high SNE level.

Our study did not include many cases, but our data showed significant correlations between the SIRS score and the anatomical and physiological severity of trauma patients. Furthermore, our data also showed the usefulness of evaluating simple vital signs in general trauma practice.

We concluded that the greater the SIRS score at the time of hospital arrival, the greater the anatomical and physiological severity of a trauma patient's condition. In addition, our results suggested that a maximum SIRS score at the time of hospital admission might be a predictor of organ dysfunction after admission. We also showed that there was a strong correlation between the fulfillment of all four SIRS criteria and an elevated SNE level.

\section{References}

1. Bone RC, Balk RA, Cerra FB, et al.: Definitions for sepsis and organ failure and guidelines for the use of innovative therapies in sepsis: the $\mathrm{ACCP} / \mathrm{SCCM}$ Consensus Conference Committee-American College of Chest Physicians/Society of Critical Care Medicine Consensus Conference Committee. Chest 1992; 101: 1644-1655.

2. Bone RC, Balk RA, Cerra FB, et al.: Definitions for sepsis and organ failure and guidelines for the use of innovative therapies in sepsis: the $\mathrm{ACCP} / \mathrm{SCCM}$ Consensus Conference Committee-American College of Chest Physicians/Society of Critical Care Medicine Consensus Conference Committee. Crit Care Med 1992; 20: 864-974.

3. Pittet D, Rangel-Frausto S, Li N, et al.: Systemic inflammatory response syndrome, sepsis, severe sepsis and septic shock: incidence, morbidities and outcomes in surgical ICU patients. Intensive Care Med 1995; 21: 302-309.

4. Jones GR, Lowes JA: The systemic inflammatory response syndrome as a predictor of bacteremia and outcome from sepsis. Q J Med 1996; 89: 515-522.

5. Bone RC: Sepsis, sepsis syndrome, and the systemic inflammatory response syndrome (SIRS): Gulliver in Laputa. JAMA 1995; 273: 155-156.

6. Sibbald WJ, Doig G, Inman KJ: Sepsis, SIRS, and infection. Intensive Care Med 1995; 21: 299-301.

7. Menger MD, Vollmar B: Systemic inflammatory response syndrome (SIRS) and sepsis in surgical patients. Intensive Care Med 1996; 22: 616-617.

8. Haga Y, Beppu T, Doi K, et al.: Systemic inflammatory response syndrome and organ dysfunction following gastrointestinal surgery. Crit Care Med 1997; 25: 1994-2000.

9. Moore EE, Moore FA, Franciose RJ, Kim FJ, Biffl WL, Banerjee A: The postischemic gut serves as a priming bed for circulating neutrophils that provoke multiple organ failure. J Trauma 1994; 37: 881-887.

10. Nystrom PO: The systemic inflammatory response syndrome: definitions and aetiology. J Antimicrob Chemother 1998; 41 Suppl A: 1-7.

11. Alberti C, Brun-Buisson C, Chevret S, et al.; European Sepsis Study Group: Systemic inflammatory response and progression to severe sepsis in critically ill infected patients. Am J Respir Crit Care Med 2005; 171: 461-468.

12. Brun-Buisson C: The epidemiology of the systemic inflammatory response. Intensive Care Med 2000; 26 Suppl 1: S64-74.

13. Marshall JC: SIRS and MODS: What is their relevance to the science and practice of intensive care? Shock 2000; 14: 586-589.

14. Rangel-Frausto MS, Pittet D, Costigan M, Hwang T, Davis CS, Wenzel RP: The natural history of the systemic inflammatory response syndrome (SIRS). A prospective study. JAMA 1995; 273: 117-123.

15. Ono S, Aosasa S, Tsujimoto H, Ueno C, Mochizuki H: Increased monocyte activation in elderly patients after surgical stress. Eur Surg Res 2001; 33: 33-38.

16. Kasai $\mathrm{T}$, Inada $\mathrm{K}$, Takakuwa $\mathrm{T}$, et al.: Antiinflammatory cytokine levels in patients with septic shock. Res Commun Mol Pathol Pharmacol 1997; 98: $34-42$.

17. Tsumura H, Ichikawa T, Hiyama E, Murakami Y, Sueda T: Systemic inflammatory response syndrome (SIRS) as a predictor of strangulated small bowel obstruction. Hepatogastroenterology 2004; 51: 13931396.

18. Claassen J, Vu A, Kreiter KT: Effect of acute physiologic derangements on outcome after subarachnoid hemorrhage. Crit Care Med 2004; 32: 832-838.

19. Napolitano LM, Ferrer T, McCarter RJ Jr, Scalea TM: Systemic inflammatory response syndrome 
score at admission independently predicts mortality and length of stay in trauma patients. J Trauma 2000; 49: 647-653.

20. Miller PR, Munn DD, Meredith JW, Chang MC: Systemic inflammatory response syndrome in the trauma intensive care unit: who is infected? J Trauma 1999; 47: 1004-1008.

21. Hoover L, Bochicchio GV, Napolitano LM, et al: Systemic inflammatory response syndrome and nosocomial infection in trauma. J Trauma 2006; 61: 310-317.

22. Castelli GP, Pognani C, Meisner M, Stuani A, Bellomi D, Sgarbi L: Procalcitonin and C-reactive protein during systemic inflammatory response syndrome, sepsis and organ dysfunction. Crit Care 2004; 8: R234-R242.

23. Shimetani N, Shimetani K, Mori M: Clinical evaluation of the measurement of serum procalcitonin: comparative study of procalcitonin and serum amyloid A protein in patients with high and low concentrations of serum C-reactive protein. Scan J Clin Lab Invest 2004; 64: 469-474.

24. Kawabata K, Hagio T, Matsuoka S: The role of neutrophil elastase in acute lung injury. Eur J Pharmacol 2002; 451: 1-10.

25. Gando S, Kameue T, Matsuda N, Hayakawa M, Hoshino H, Kato H: Serial changes in neutrophilendothelial activation markers during the course of sepsis associated with disseminated intravascular coagulation. Thromb Res 2005; 116: 91-100.

(Received, December 21, 2009)

(Accepted, January 22, 2010) 